\title{
Ingeniería Naturalística para prevención de riesgos en dos taludes del sur de Quito
}

\section{(Naturalistic Engineering for risk prevention in two slopes in southern Quito)}

\author{
Anita Argüello Mejía ${ }^{1}$, Jimena Cumbicos Ortiz ${ }^{1}$, Claudia Mosquera Cañizares ${ }^{1}$, Jimmy \\ Vicente-Reyes ${ }^{1}$
}

\begin{abstract}
Resumen:
La Ingeniería Naturalística es una ciencia moderna técnico-científica que combina la Ingeniería Civil, Ambiental y Geotécnica. Estudia y utiliza materiales de construcción, plantas y materiales orgánicos y sintéticos para sostener taludes. En San Luis de Chillogallo y El Recreo, sectores ubicados al sur de Quito, se han realizado obras de control de erosión, contención y recuperación ambiental con el objetivo de generar estrategias y capacidades de estimación y reducción de riesgos de desastres en dos administraciones zonales de la ciudad. Para implementar las obras se intervino el terreno, se limpió la materia orgánica e inorgánica, se removió las partes inestables de la pendiente y se remodeló a través de excusión y recolocación de tierra. Posteriormente, de acuerdo a la pendiente de cada talud se escogió y diseñó la técnica utilizada. En el sector de San Luis de Chillogallo se utilizaron las técnicas Entramado de doble pared y Entramado latino. En el sector de El Recreo se utilizaron las técnicas Emparrillado vivo y Entramado latino. Para las obras implementadas se utilizaron plantas como: aliso, Alnus glutinosa; árbol de papel, Polylepis sp.; chilca, Baccharis latifolia; lechero, Euphorbia lactiflua y tilo, Tilia platyphyllos scopoli, las cuales han tenido un rápido crecimiento y se han adaptado exitosamente a los dos taludes intervenidos.
\end{abstract}

Palabras claves: Ingeniería Naturalística; taludes; riesgos; técnicas de remediación; construcción.

\begin{abstract}
:
Naturalistic engineering is a technical-scientific modern science cobining civil, environmental and geotechnical engineerings. It studies and uses building materials, plants, organic and synthetic materials for holding slopes. San Luis de Chillogallo and El Recreo are located in the South of Quito, where two projects for erosion control, containment and environmental recovery, have been implemented. These are pilot interventions that allow applying strategies and capabilities of estimation and reduction of risks from disasters. To implement the works, the ground was shifted, the organic and inorganic matter was wiped out, and unstable parts of the slope were removed, reshaping the slope through land exclusion and relocation. Subsequently, depending on the shape of each slope, specific techniques where designed and implemented. Double Wall Crib and Latin Triangular Branching techniques were used in San Luis de Chillogallo. Live Grating and Latino Triangular Branching techniques were used in El Recreo. Plants such as: Alder, Alnus glutinosa; paper tree, Polylepis sp.; chilca, Baccharis latifolia; lechero Euphorbia lactiflua and Tilo, Tilia platyphyllos; have been used in these projects. These plants are fast growing species and they have adapted successfully on the two slopes intervened.
\end{abstract}

Keywords: Naturalistic engineering; slopes; risks; remediation techniques; construction.

\footnotetext{
${ }^{1}$ Universidad Tecnológica Equinoccial, Facultad de Ciencias de la Ingeniería, Quito - Ecuador (aarguello@ute.edu.ec)
} 


\section{Introducción}

La búsqueda de nuevas técnicas para solucionar problemas ambientales, especialmente aquellos producidos por fenómenos meteorológicos como intensas precipitaciones y causas antrópicas como construcciones civiles, han sido un problema continuo. Actualmente, se está utilizando una nueva técnica conocida como "Ingeniería Naturalística" (IN) la cual está fundamentada en utilizar plantas que cumplan con características especiales de resistencia para sostener taludes y que de esta manera se pueda proteger a las personas y evitar derrumbes en vías, riveras, túneles.

El término de Ingeniería Naturalística se refiere a "una disciplina técnico-científica que estudia las modalidades de uso, como materiales de construcción en intervenciones de control de erosión, de contención y recuperación ambiental, de plantas vivas, de partes de plantas o de asociaciones vegetales, a menudo en combinación con materiales orgánicos muertos (madera, etc.) y como materiales no orgánicos naturales (piedras, tierra, etc.) y sintéticos (hierro, plástica, etc.)" (Crocetti, Ferrari, Petrone, \& AIPIN, 2012).

La IN se conoce también como "Ingeniería Biológica" y cumple una función hidrogeológica de consolidación del terreno a través de la cobertura del mismo, permitiendo la protección del suelo de la acción de la erosión y permitiendo la recuperación ambiental mediante el cambio en el valor paisajístico en armonía con el ecosistema (Petrone, 2013).

De IN ya se empezó a hablar a finales de 1800, cuando en Europa se empezó la difusión de técnicas de manejo forestal. Fueron sobre todo los cortos tiempos de realización y la economía de las obras (normalmente los materiales se recuperan directamente en el lugar en el cual se realiza la intervención), que aseguraron el éxito y la rápida difusión en otros ámbitos de aplicación (Petrone, 2013).

El propósito de la misma es poner en buenas condiciones de seguridad taludes, carreteras y edificios. Existen varias ventajas al utilizar estas técnicas como costos relativamente bajos, aumento de la biodiversidad, conservación y mejoramiento del paisaje, crear un hábitat para la fauna. Las raíces favorecen la depuración de impurezas presentes en el agua, por ejemplo, asimilando los excesos de sustancias orgánicas y absorbiendo también metales pesados u otras sustancias químicas.

Se debe tener en cuenta que también pueden presentarse ciertas limitantes que afectan dicha técnica tales como la necesidad de un mantenimiento regular y en ocasiones los resultados no son inmediatos.

El objetivo del presente artículo es presentar las técnicas de IN aplicadas en dos taludes de las Administraciones Zonales Eloy Alfaro y Quitumbe del MDMQ como parte del proyecto "Dos zonas administrativas fortalecen sus capacidades de reducción del riesgo de desastres, ejecutando 
mecanismos piloto de Ingeniería Naturalística en Quito", ejecutado por el Centro Regionale d'Intervento per la Cooperazione CRIC, y orientado a la protección de zonas vulnerables y a ser un aporte a la reflexión para la conservación de los recursos naturales como mecanismo para la reducción del riesgo.

El sector Sur de la ciudad de Quito es conocido por la gran concentración demográfica. Antiguamente Chillogallo y El Recreo eran zonas muy rurales en donde no existían construcciones civiles. Actualmente, debido a las invasiones de terrenos, se ha generado una amplia gama de construcciones, lo cual ha ocasionado el movimiento o fractura del terreno, dando como resultado deslizamientos o caída de materiales de taludes, afectando negativamente a los pobladores de esta zona. El estudio tuvo como objetivo realizar el monitoreo mensual de las obras de IN en donde se utilizaron técnicas de entramado de madera a doble pared y emparrillado vivo en los sectores de San Luis de Chillogallo y El Recreo en la ciudad de Quito, respectivamente. Las técnicas utilizadas además de dar estabilidad al terreno, son técnicas estéticas y creativas porque generan obras que tienen una belleza natural.

Para la realización del estudio, se tomó en cuenta la tabulación de los datos obtenidos en los monitoreos mensuales, para realizar comparaciones y desarrollar recomendaciones de acuerdo al comportamiento de las plantas utilizadas para sostener taludes en los sectores antes mencionados.

\section{Materiales y métodos}

Para el diseño y elaboración de Ingeniería Naturalística (IN) se realiza un estudio del sitio de intervención en conjunto con un equipo de técnicos y posteriormente se realizan análisis topográficos, geomorfológicos, litológicos, geotécnicos, hidrológicos y de vegetación.

Además, para la elaboración del estudio se debe contar con documentos de planificación elaborados con la información existente de la zona a ser intervenida y muestras fotográficas. En los sectores donde se implementan las obras de IN, se debe contar con estudios previos del terreno. La inspección se realiza con la utilización de instrumentos como: cinta métrica, clinómetro, GPS, palos de madera, manguera de hule con agua para medir la pendiente de los taludes, cámara, cuaderno y mapa de la zona, posteriormente se mide el área y establece las intervenciones a ser realizadas. Se aplican las técnicas entramado de madera a doble pared y emparrillado vivo en las dos obras implementadas de IN de El Recreo (Administración Zonal Eloy Alfaro) y San Luis de Chillogallo (Administración Zonal Quitumbe) respectivamente. 


\subsection{Talud de San Luis de Chillogallo barrio perteneciente a la Administración Zonal Quitumbe, MDMQ}

\section{Técnica de Entramado de madera a doble pared y Entramado Latino}

Se implementa al pie del talud para evitar los movimientos del terreno y mejorar el drenaje. Las raíces de las estacas funcionan como sostén de la estructura cuando esta se deshaga y drenan la pendiente. El entramado latino se utiliza en la base para realizar un sustento a la obra de IN como se puede observar en la Figura 1.
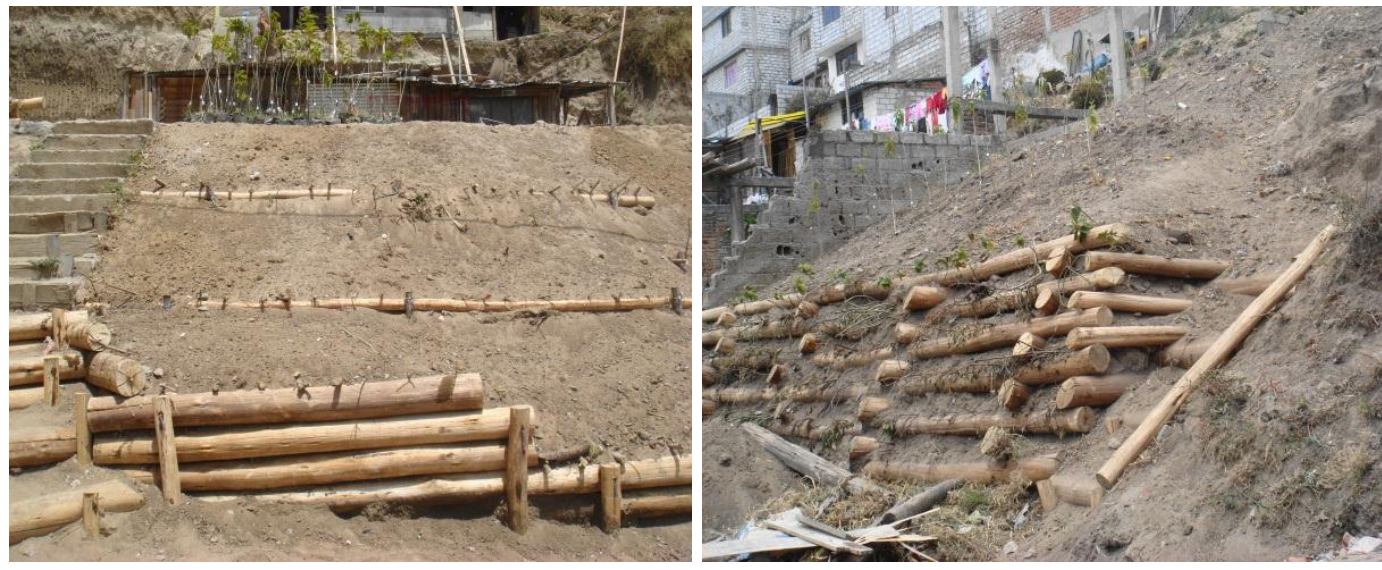

Figura 1. Técnica de entramado doble pared y latino

\section{Procedimientos de implementación}

- Se limpia el sitio a ser intervenido y se quita las partes inestables,

- Se realiza la base de la obra excavando a una profundidad similar al diámetro de los palos, esta base debe ser lisa e inclinada en sentido contraria a la pendiente,

- Se colocan los palos en varios niveles unidos de manera entrecruzada formando una caja en el terreno.

- Se colocan las estacas, estas deben llegar al fondo y sobresalir de 10-20 cm, la densidad es igual a 4 estacas/metro y es necesario destacar que es fundamental utilizar especies vegetales del lugar y se coloca chamba en casos requeridos. En los monitoreos se realizará la medición de diámetro y longitud de las estacas y luego de los brotes que tengan las mismas.

- La estructura tiene dos paredes paralelas que están en contacto con el terreno: una externa (frontal) y otra interna. Estas paredes se inclinan máximo a 60 grados con respecto a la horizontal, la profundidad y altura máxima es de 2.25 metros para garantizar un suficiente aporte de agua lluvia para el crecimiento de las plantas y soporte del talud. 
Tabla 1. Plantas utilizadas

\begin{tabular}{|l|l|}
\hline NOMBRE VULGAR & NOMBRE CIENTÍFICO \\
\hline Aliso & Alnus glutinos. \\
\hline Árbol de papel & Polylepis sp \\
\hline Chilca & Baccharis latifolia \\
\hline Lechero & Euphorbia lactiflua \\
\hline Pusupato & Tidllansia sp. \\
\hline Tilo & Tilia platyphyllos scopoli \\
\hline
\end{tabular}

Ventajas:

Rápida consolidación y construcción

Recrea áreas naturales y paisaje

Fácil obtención de estacas aledañas a - Reposición de estacas o tierra en algunos la obra
Desventajas:

- No alcanza grandes alturas

- Riego en periodos críticos casos.

\subsection{Talud de EI Recreo barrio perteneciente a la Administración Zonal EI Recreo, MDMQ}

\section{Técnica de Emparrillado vivo}

Estructura elaborada a partir de palos de madera colocados horizontalmente y verticalmente, forma una red que sostiene al terreno y las plantas. Su función es de sostener y contener el material relleno, las raíces de las estacas consolidaran la ladera y favorecerán el drenaje. El entramado latino se utiliza en la base para realizar un sustento a la obra de IN como se puede observar en la Figura 2.
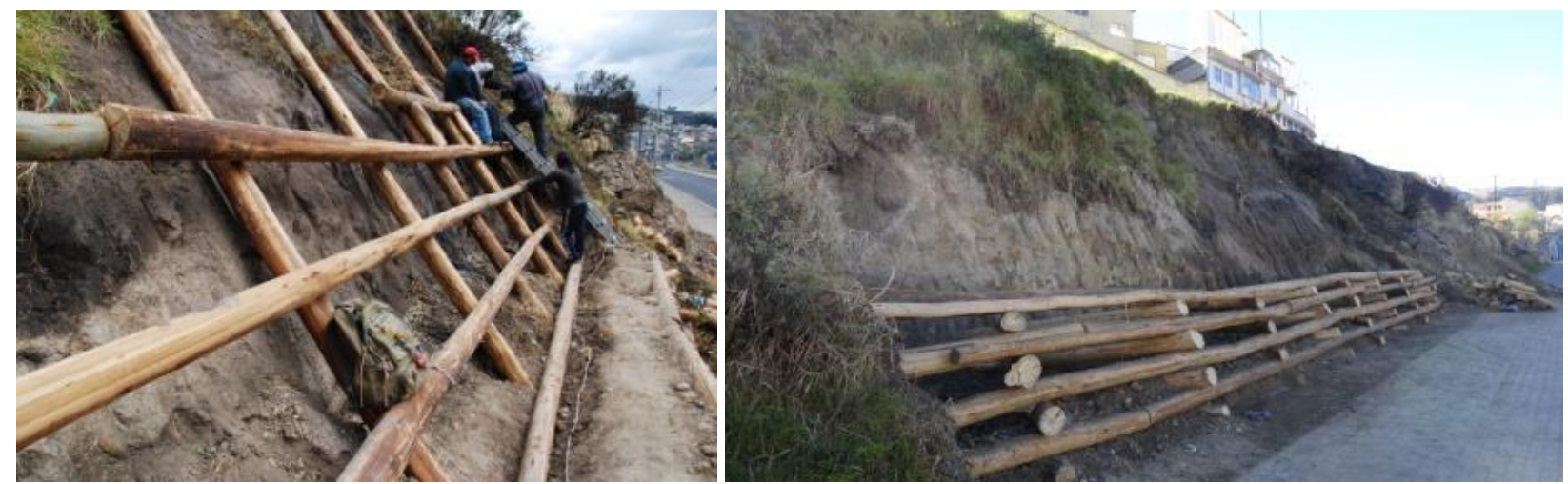

Figura 2. Técnica Emparrillado vivo y entramado latino Fuente: Terranova-CRIC (2012)

\section{Procedimientos de implementación}

- Se limpia el sitio a ser intervenido y se quita las partes inestables,

- Se realiza la base de la obra excavando una zanja profunda similar al doble del diámetro de los palos,

- Se colocan los palos en varios niveles unidos de manera horizontal y vertical en hileras sobrepuestas que sirven de base para la obra distanciada máximo de 2 metros, 
- Se coloca las estacas, estas deben llegar al fondo y sobresalir de $10-20 \mathrm{~cm}$, destacar que es fundamental utilizar especies vegetales del lugar y se coloca chamba en casos requeridos. En los monitoreos se realizará la medición de diámetro y longitud de las estacas y luego de los brotes que tengan las mismas.

- La estructura toma forma de malla cuadrangular o rectangular. Esta técnica es utilizada para el control de fuertes erosiones, es empleada en riveras de ríos y laderas. La inclinación máxima es de 55 grados y puede alcanzar alturas máximas de hasta 20 metros.

Plantas utilizadas

Descrito en la Tabla 2.

Tabla 2. Plantas utilizadas

\begin{tabular}{|l|l|}
\hline NOMBRE VULGAR & NOMBRE CIENTÍFICO \\
\hline Aliso & Alnus glutinos. \\
\hline Árbol de papel & Polylepis $s p$ \\
\hline Chilca & Baccharis latifolia \\
\hline
\end{tabular}

Ventajas:

- Posible de realizar en espacios estrechos

- Alcanza inclinaciones muy elevadas

- Rápida estabilización y drenaje bueno

- Buena vista paisajística y ambiental
Desventajas:

- Construcción lenta

- Sensible al deterioro

- No aplicable en caso de presencia de rocas

Al momento de colocar las plantas, se lleva el registro del sitio donde se coloca la estaca, el diámetro y la longitud de la estaca y se contabiliza las estacas utilizadas en cada obra. Durante el periodo Septiembre 2012 - 2013, mensualmente se realiza el monitoreo de las obras para determinar el comportamiento de las estacas que fueron colocadas y en cada talud, posteriormente se contabiliza también la vitalidad los brotes de las estacas si estas poseen.

\section{Resultados}

\subsection{San Luis de Chillogallo}

De acuerdo a las figuras 3 y 4 se presentan las medias del diámetro y de la longitud de las estacas utilizadas en San Luis de Chillogallo donde se puede observar la variación de crecimiento; respecto al diámetro de las estacas en la Figura 3 donde se alcanza un aumento del mismo con un promedio entre 0,93 a 2,03 cm con regularidad durante todo el año, en todas las especies, con una disminución en el mes de julio, donde existió un descuido por parte de los moradores del barrio, perdiéndose algunas estacas y encontrándose inclusive basura en la obra de contención del talud. 


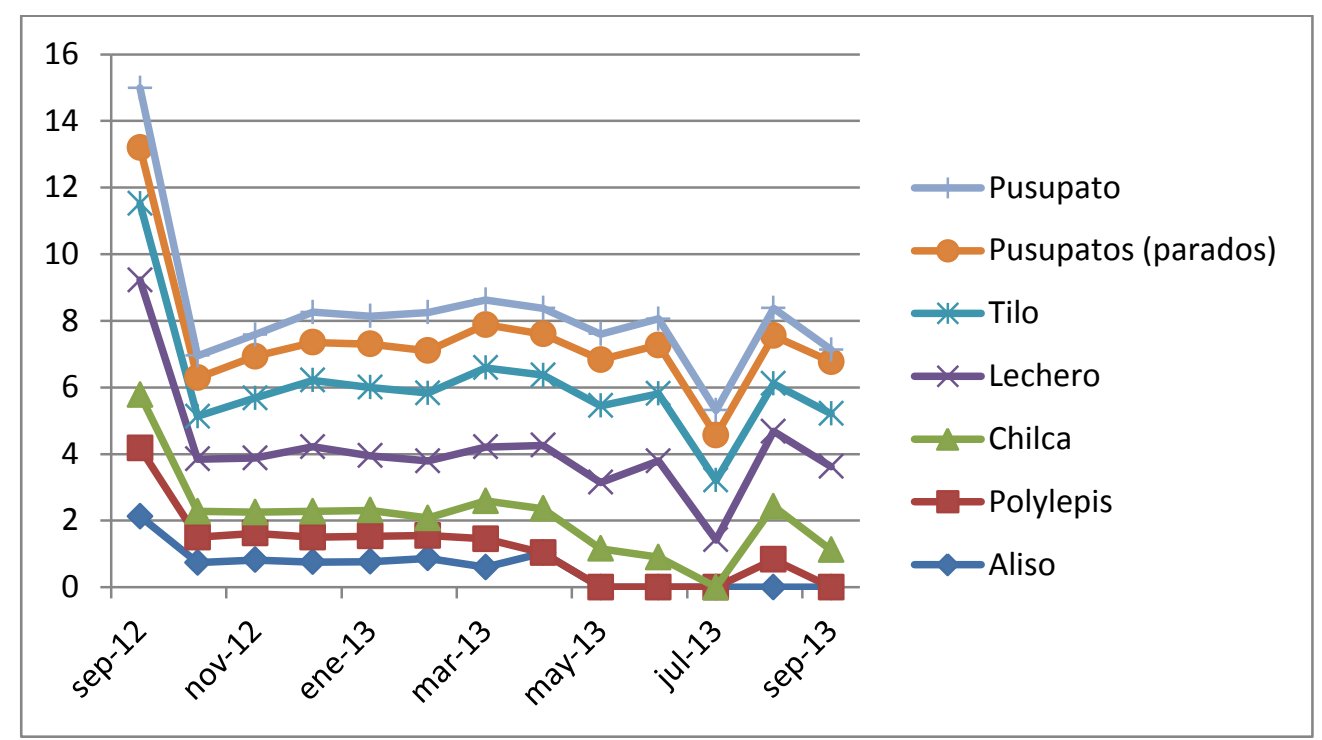

Figura 3. Diámetro en $\mathrm{cm}$ de las estacas de las plantas utilizadas

En la Figura 4, con respecto a la longitud de las estacas se observa un promedio de crecimiento entre 0,32 a 1,03 durante el año de todas las especies sembradas. Sin embargo se observa un crecimiento acumulado mejor en los pusupatos con un crecimiento regular de $1 \mathrm{~cm}$ por mes.

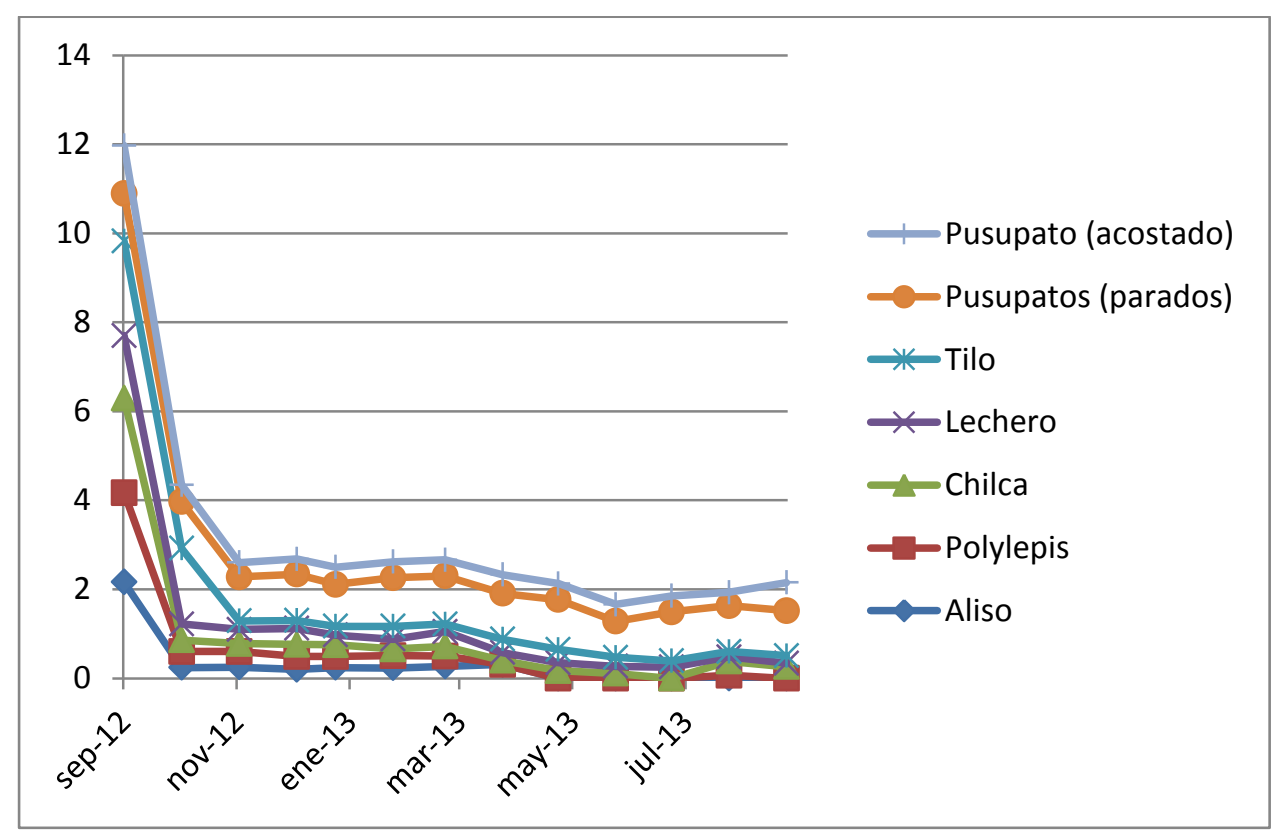

Figura 4. Longitud en $\mathrm{cm}$ de las estacas de las plantas utilizadas

De acuerdo a la Figuras 5 se observa el crecimiento del diámetro de los brotes entre 0,5 a 1,03 cm como promedio en todos los meses, teniendo una mejor adaptación la Chilca con un crecimiento acumulado mayor con más de $1 \mathrm{~cm}$ en promedio durante todo el año. El lechero y el pusupato tienen también una buena adaptación presentando un promedio de más de 0,70 cm. 


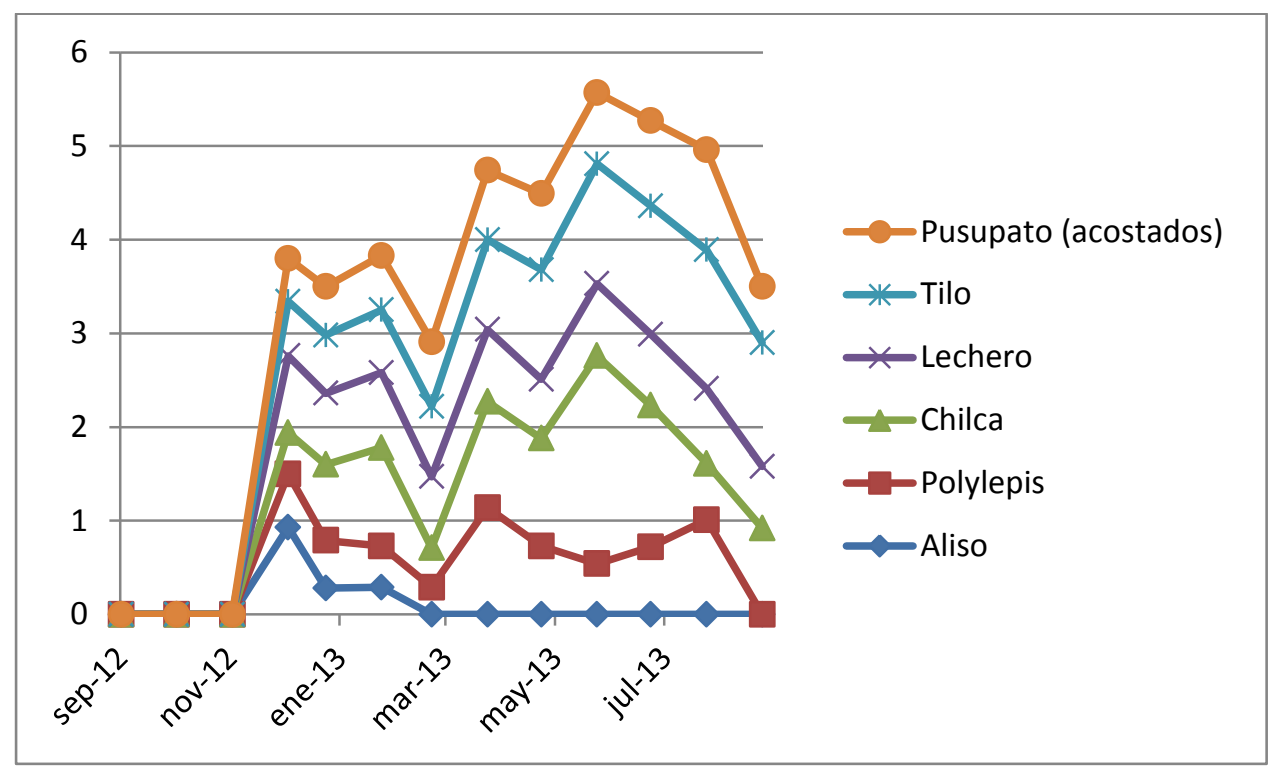

Figura 5. Diámetro en $\mathrm{cm}$ de los brotes de las plantas utilizadas

En la Figura 6 se observa que el crecimiento promedio de los brotes en longitud, el cual oscila entre 0,31 a 0,81 cm durante el año, con excepción del aliso y el Polylepis que decaen en el crecimiento en los seis últimos meses ya que algunas estacas se pierden debido a la falta de cuidado por parte de los pobladores.

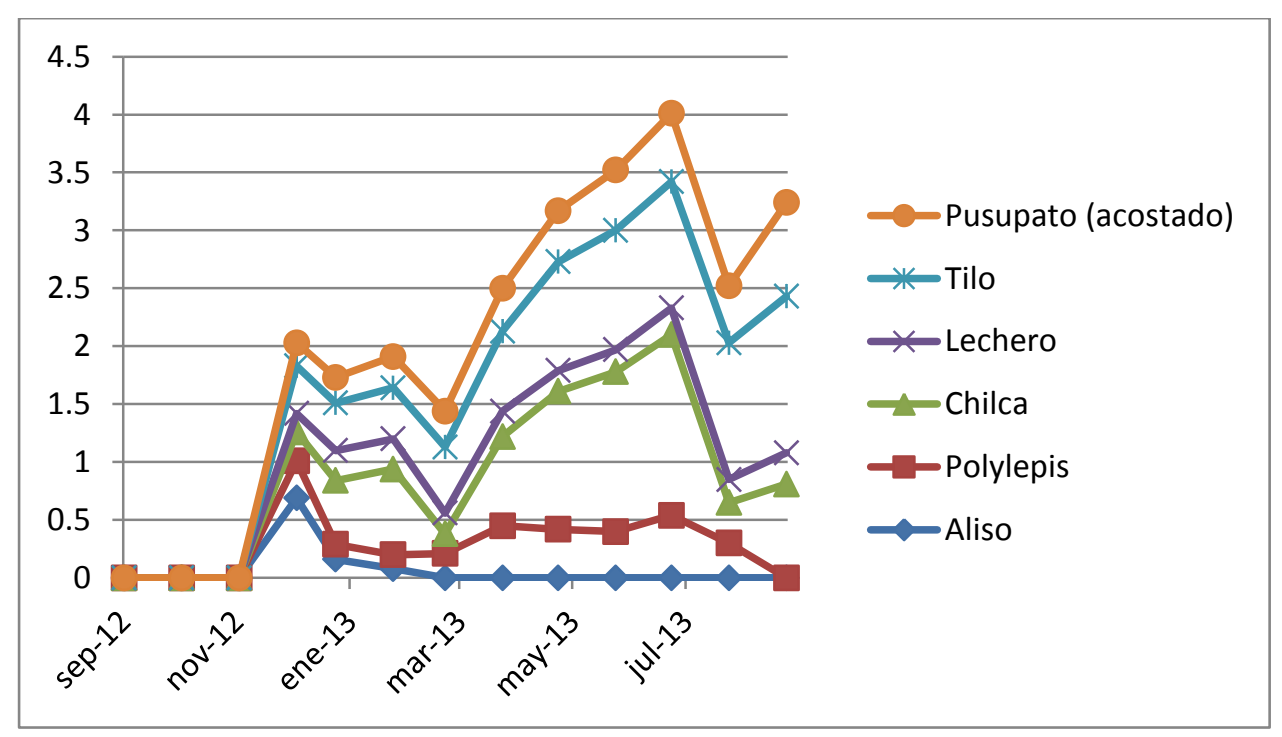

Figura 6. Longitud de los brotes de las plantas utilizadas

\subsection{EI Recreo}

De acuerdo a las figuras 7 y 8 se observa que el crecimiento promedio de las estacas es de 0,5 a $1,2 \mathrm{~cm}$ por mes en lo que respecta al diámetro de las mismas, con un descenso en su desarrollo de las estacas en diciembre 2012 y abril y agosto de 2013. 


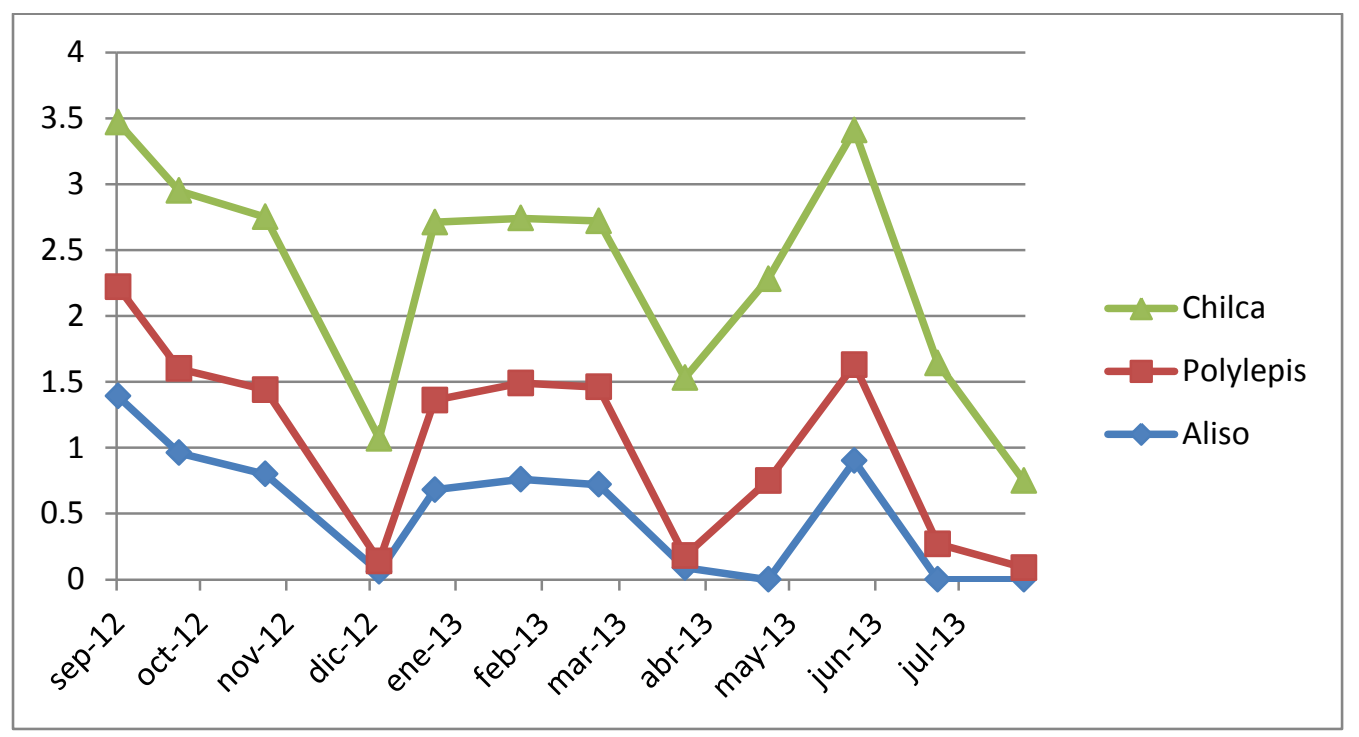

Figura 7. Diámetros estacas de plantas utilizadas

Respecto a la longitud no hay un crecimiento adecuado en las tres especies, siendo de 0,26 a 0,35 lo que disminuye la posibilidad del establecimiento de las mismas.

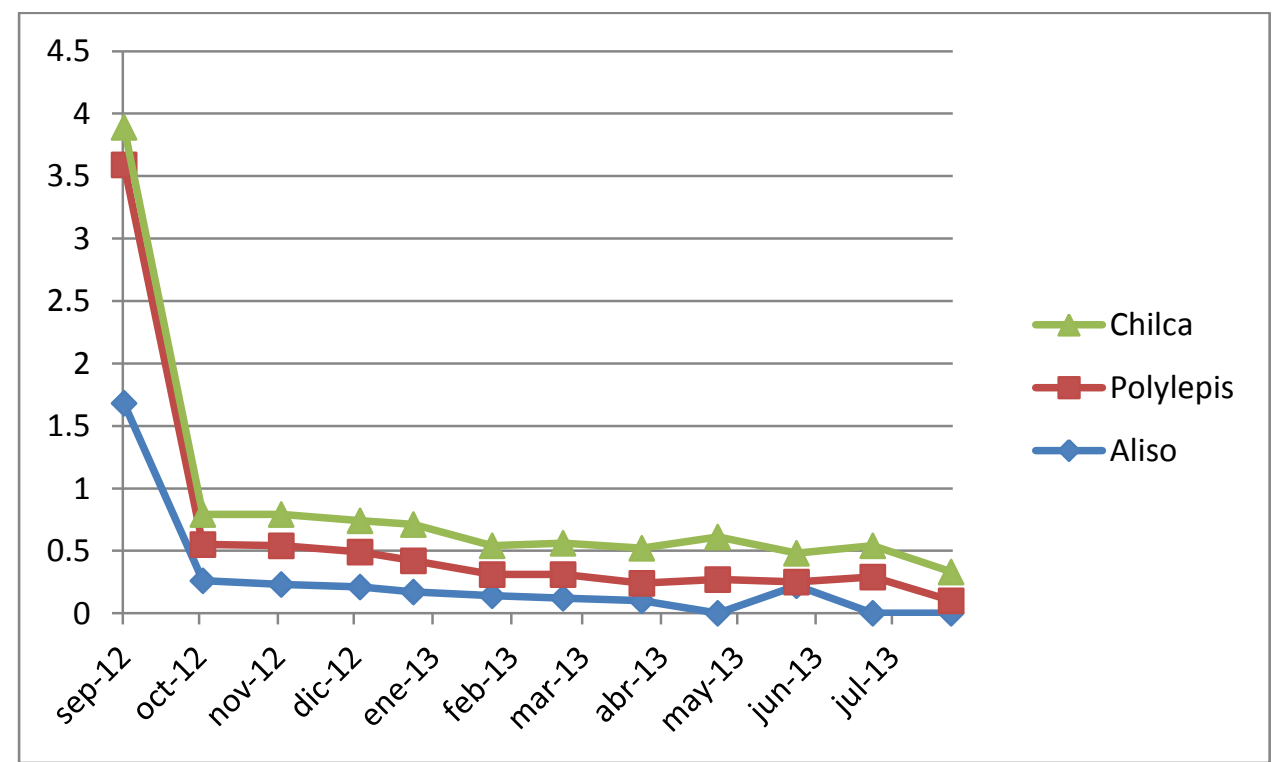

Figura 8. Longitudes de estacas de plantas utilizadas

De acuerdo a la Figura 9 el diámetro de los brotes tiene un promedio de crecimiento de 0,31 a 0,48 , lo que no se considera significativo puesto que no permite el establecimiento de las especies sembradas. Se debe notar también que tiene relación directa con el diámetro de las estacas con los descensos en crecimiento en los mismos meses mencionados anteriormente. Los brotes tienen dificultad de prologarse con excepción de la chilca que tiene un crecimiento mensual de 0,5 $\mathrm{cm}$. 


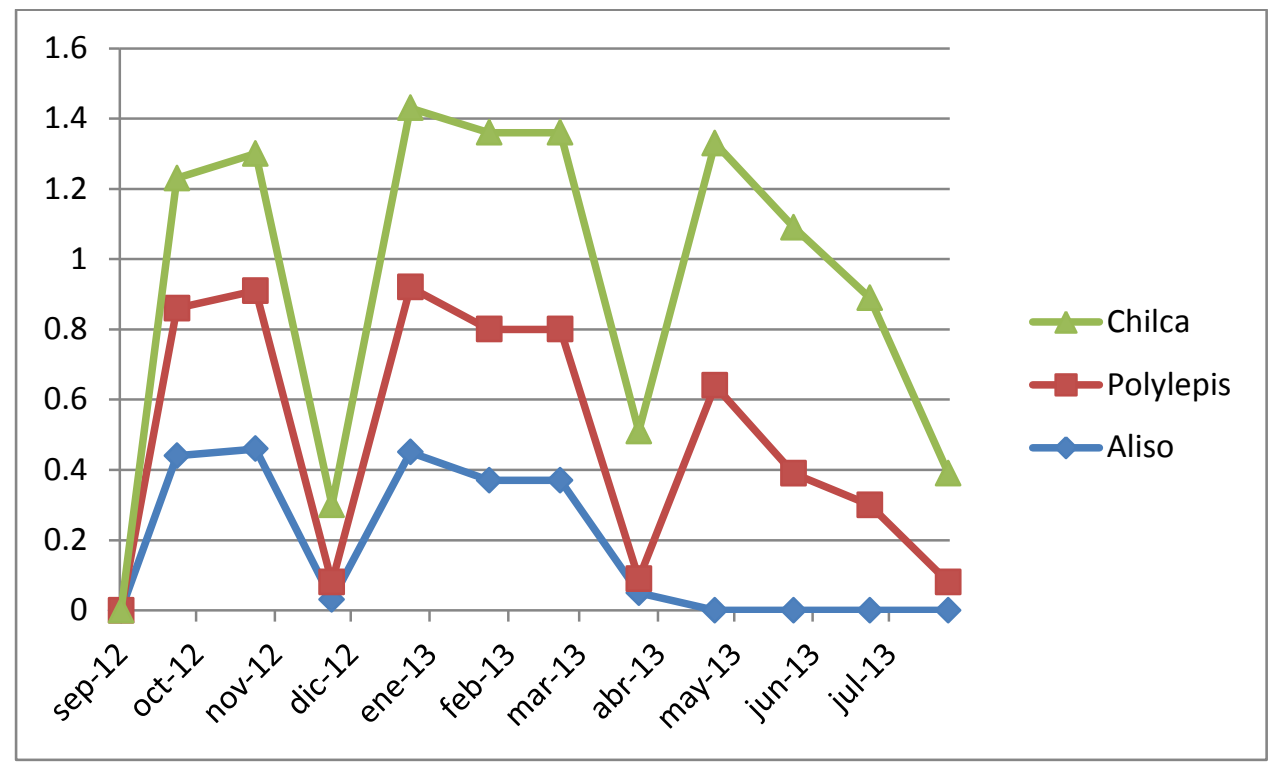

Figura 9: diámetros brotes de las plantas utilizadas

Conforme a la Figura 10, se observa que el promedio de crecimiento en longitud de los brotes es mínimo ya que va de 0,09 a $0,10 \mathrm{~cm}$, con excepción de la chilca que llega a un crecimiento promedio de $0,44 \mathrm{~cm}$ y permanece mejor establecida.

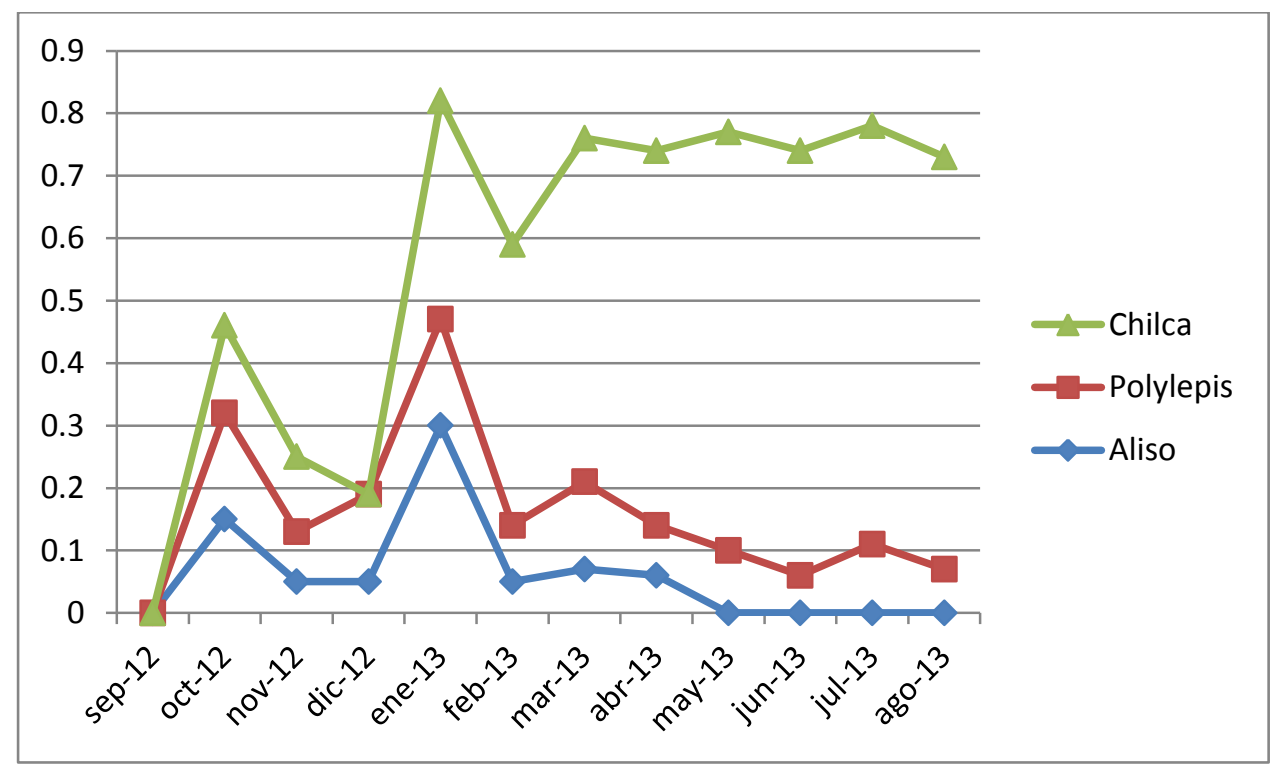

Figura 10: longitudes de brotes de plantas utilizadas

\section{Discusión}

De acuerdo a los monitoreos que se han realizado, se puede observar que la vitalidad de las plantas es óptima, dando como resultado el sostén del talud. Lo que significa que la Ingeniería Naturalística es sostenible y realmente disminuye el riesgo de la erosión y caída de taludes por intervención antrópicas. El monitoreo durante todo el año ha permitido ver el comportamiento de las plantas y determinar cuáles son óptimas para este tipo de terreno. En las obras de Ingeniería Naturalística las plantas son instrumentos vivos que ayudan a sostener taludes y brindar estética al lugar en donde se ha implementado este tipo de obras. 
La vitalidad de las plantas, utilizadas para sostener taludes en San Luis de Chillogallo y El Recreo, es muy buena porque estas presentan brotes y tienen una coloración muy saludable. Además no se observa que plagas las estén afectando. Es necesario realizar una limpieza en los lugares del monitoreo porque existió en algunos lugares acumulación de basura.

En algunos meses se observó el crecimiento no constante de las plantas debido a los cambios de clima (falta de lluvias) y descuido por parte de los pobladores aledaños a las obras y de las instituciones participantes. En los meses de Julio a Septiembre de 2013, existe disminución en el número de plantas sembradas debido a que el descuido en los sectores intervenidos.

Finalmente, se debe indicar que se ha cumplido un año de las obras de Ingeniería Naturalística en San Luis de Chillogallo e indicar que las especies se han mantenido y han tenido un rápido crecimiento y se han adaptado exitosamente a los dos taludes intervenidos.

El crecimiento es constante y de mejor adaptación del pusupato y la chilca en San Luis de Chillogallo como en El Recreo.

\section{Conclusiones y Recomendaciones}

En las obras de IN las plantas son instrumentos vivos que ayudan a sostener taludes y brindar estética al lugar en donde se ha implementado este tipo de obras. Existen varias ventajas al utilizar estas técnicas como costos bajos, aumento de la biodiversidad, conservación y mejoramiento del paisaje, crean un hábitat para la fauna, sus raíces favorecen la depuración de impurezas presentes en el agua asimilando los excesos de sustancias orgánicas y absorbiendo también metales pesados u otras sustancias químicas, etc.

De acuerdo a los monitoreos que se han realizado, se puede observar que la vitalidad de las plantas es buena, sin embargo, el crecimiento es lento tanto en estacas como en los brotes de las mismas. A pesar de ello, el establecimiento de especies como el pusupato y la chilca a permitido mantener los taludes estables indistintamente de las técnicas empleadas, dando como resultado el sostén del talud. Lo que significa que las técnicas de han permitido disminuir el riesgo de la erosión y caída de taludes en las dos obras realizadas.

Durante los primeros meses de monitoreo los resultados demuestran que el crecimiento es más sostenido permitiendo el establecimiento de las estacas y el desarrollo de los brotes con coloración saludable en los dos taludes en San Luis de Chillogallo y El Recreo.

No se observó la presencia de plagas que puedan afectar las obras implementadas, pero se observó la disminución e incluso desaparición de algunas especies en las obras, como el Aliso y Polylepis, dada la negligencia de los pobladores encargados de la poda de las plantas 
contribuyendo a su desaparición. Además de que por la exposición de las obras basura lo que ha facilitado la inhibición de las plantas.

Las plantas que se han adaptado a las condiciones climáticas de los sectores y a las características físicas del lugar continuaron con su función de sostener el talud entre ellas se encuentran especies como la Chilca y en el San Luis también el Tilo.

Tanto las administraciones zonales en los sectores intervenidos, la falta de cuidado de los moradores y de las autoridades corresponsables del proyecto, evidencio disminución en el número de plantas sembradas debido al descuido, lo que ha provocado que las especies disminuyan su crecimiento o desaparezcan.

Tras un año de las dos obras piloto en Quito, se puede concluir que se realizó un gran esfuerzo por mantener las obras en buen estado y se pudo comprobar que esta técnica es factible y ecológicamente viable y sostenible.

Se deberían realizar mingas comunitarias cada quince días, las cuales evitarán que las especies sembradas sean cubiertas por plantas y plagas, además ayudaran a evitar que vectores externos al talud como basura, materiales de construcción, entre otros, se sitúen en el mismo generando malos olores o alterando la vegetación y el cuerpo del talud.

Se debe realizar limpiezas trimestrales en los lugares del monitoreo puesto que es complicado realizar los monitores por la acumulación de basura y plantas invasoras.

Se sugiere que las personas responsables del proyecto, es decir, la Administración Zonal de Quitumbe y El Recreo, y los moradores de los sectores intervenidos entablen comunicación continua para la limpieza, mantenimiento y cuidado de las obras.

Se recomienda dar mayor información a la población sobre el uso de este tipo de técnicas que permiten conservar los recursos naturales manteniendo un paisaje agradable a la vista.

Se recomienda a las Administraciones Zonales replicar esta técnica en lugares donde se den con mayor frecuencia deslizamientos, derrumbes y erosión del suelo, ya que la técnica no es compleja de construir ni implica costos altos para la población.

\section{Bibliografía}

AQUANEA (2011). Ingeniería Naturalística. En línea, recuperado el 22 de Septiembre del 2013, disponible en,http://www.aquanea.com/es/tecniques:Cos/menulateral:tecniques:naturalistica.

Crocetti, C., Ferrari, R., Petrone, A., \& AIPIN, D. . (2012). Manual de Ingeniería Naturalística. Quito: CRIC. 
Cumbicos, J., Mosquera, C., Vicente, J., Zuleta, D. (2013). Informe del comportamiento de las especies vegetales sembradas en los barrios de El Recreo y San Luis de Chillogallo. Universidad Tecnológica Equinoccial - CRIC. Quito, Ecuador.

Desaprender (2008). Reducción de riesgo de desastres naturales en el sur de Manabí. La Ingeniería Naturalística: Practicas ecológicamente sostenibles y de bajo costo para la reducción de riesgos frente a desastres. En línea, recuperado el 20 de Septiembre de 2012, de, http://www.desaprender.org/tools/la-ingenieria-naturalistica-practicas-ecologicamentesostenibles-y-de-bajo-costo-para-la-reduccion-de-riesgos-frente-a-desastres

Fundación Terranueva-CRIC. (2012). Curso de Capacitación en Técnicas de Ingeniería Naturalística. Qué es, Principales técnicas, Ejemplos de técnicas, Verificaciones de estabilidad de Ingeniería Naturalística. Universidad Tecnológica Equinoccial. Quito, Ecuador.

Fundación Terranueva - CRIC (2012). Manual de Ingeniería Naturalística. Quito, Ecuador. 2012.

Petrone, A. y. (2013). La Ingenierí Naturalística. Técnicas Apropiadas y Sostenibles para la Reducción de la Vulnerabilidad en el Territorio. Recuperado el 13 de 10 de 2013, de www.ideassonline.org: http://www.ideassonline.org/public/pdf/br_37_79.pdf

Quintana, Y., Petrone, A., Preti, F. (2009). Capitalización de la experiencia de Ingeniería Naturalística. CRIC - Fundación Terranueva. Manabí, Ecuador. 
ANEXOS

San Luis de Chillogallo

\begin{tabular}{|c|c|c|c|c|c|c|c|c|c|c|c|c|c|c|c|c|c|c|c|c|c|c|c|c|c|c|}
\hline \multirow{3}{*}{ Mes } & \multicolumn{4}{|c|}{ Aliso } & \multicolumn{4}{|c|}{ Polylepis } & \multicolumn{4}{|c|}{ Chilca } & \multicolumn{4}{|c|}{ Lechero } & \multicolumn{4}{|c|}{ Tilo } & \multirow{2}{*}{\multicolumn{2}{|c|}{$\begin{array}{c}\begin{array}{c}\text { Pusupatos } \\
\text { (parados) }\end{array} \\
\text { Estaca }\end{array}$}} & \multicolumn{4}{|c|}{$\begin{array}{l}\text { Pusupato } \\
\text { (acostados) }\end{array}$} \\
\hline & \multicolumn{2}{|c|}{ Estaca } & \multicolumn{2}{|c|}{ Brote } & \multicolumn{2}{|c|}{ Estaca } & \multicolumn{2}{|c|}{ Brote } & \multicolumn{2}{|c|}{ Estaca } & \multicolumn{2}{|c|}{ Brote } & \multicolumn{2}{|c|}{ Estaca } & \multicolumn{2}{|c|}{ Brote } & \multicolumn{2}{|c|}{ Estaca } & \multicolumn{2}{|c|}{ Brote } & & & \multicolumn{2}{|c|}{ Estaca } & \multicolumn{2}{|c|}{ Brote } \\
\hline & $\mathrm{D}$ & $\mathrm{L}$ & $\mathrm{D}$ & $\mathrm{L}$ & $\mathrm{D}$ & $\mathrm{L}$ & $\mathrm{D}$ & $\mathrm{L}$ & $\mathrm{D}$ & $\mathrm{L}$ & $\mathrm{D}$ & $\mathrm{L}$ & $\mathrm{D}$ & $\mathrm{L}$ & $\mathrm{D}$ & $\mathrm{L}$ & $\mathrm{D}$ & $\mathrm{L}$ & $\mathrm{D}$ & $\mathrm{L}$ & $\mathrm{D}$ & $\mathrm{L}$ & $\mathrm{D}$ & $\mathrm{L}$ & $\mathrm{D}$ & $\mathrm{L}$ \\
\hline $09 / 12$ & 2.13 & 2.16 & & & 2.05 & 2.01 & & & 1.60 & 2.12 & & & 3.44 & 1.41 & & & 2.30 & 2.12 & & & 1.68 & 1.07 & 1.79 & 1.08 & & \\
\hline $10 / 12$ & 0.74 & 0.24 & & & 0.76 & 0.36 & & & 0.78 & 0.26 & & & 1.57 & 0.36 & & & 1.28 & 1.70 & & & 1.16 & 1.05 & 0.66 & 0.38 & & \\
\hline $11 / 12$ & 0.82 & 0.25 & & & 0.80 & 0.35 & & & 0.63 & 0.18 & & & 1.63 & 0.32 & & & 1.80 & 0.19 & & & 1.25 & 0.99 & 0.65 & 0.32 & & \\
\hline $12 / 12$ & 0.76 & 0.20 & 0.93 & 0.69 & 0.74 & 0.29 & 0.57 & 0.32 & 0.78 & 0.27 & 0.44 & 0.25 & 1.94 & 0.36 & 0.82 & 0.16 & 1.99 & 0.18 & 0.58 & 0.41 & 1.14 & 1.04 & 0.91 & 0.34 & 0.46 & 0.20 \\
\hline $01 / 13$ & 0.77 & 0.24 & 0.28 & 0.16 & 0.76 & 0.25 & 0.51 & 0.13 & 0.78 & 0.26 & 0.81 & 0.55 & 1.64 & 0.22 & 0.76 & 0.26 & 2.05 & 0.20 & 0.62 & 0.41 & 1.30 & 0.94 & 0.84 & 0.39 & 0.52 & 0.22 \\
\hline $02 / 13$ & 0.87 & 0.23 & 0.29 & 0.08 & 0.68 & 0.28 & 0.44 & 0.12 & 0.53 & 0.15 & 1.05 & 0.74 & 1.71 & 0.22 & 0.80 & 0.26 & 2.04 & 0.29 & 0.67 & 0.44 & 1.27 & 1.09 & 1.15 & 0.36 & 0.58 & 0.27 \\
\hline $03 / 13$ & 0.60 & 0.27 & & & 0.85 & 0.23 & 0.29 & 0.21 & 1.14 & 0.22 & 0.42 & 0.17 & 1.62 & 0.33 & 0.76 & 0.18 & 2.38 & 0.17 & 0.75 & 0.57 & 1.29 & 1.08 & 0.75 & 0.36 & 0.69 & 0.31 \\
\hline $04 / 13$ & 1.03 & 0.31 & & & & & 1.14 & 0.45 & 1.33 & 0.09 & 1.13 & 0.77 & 1.90 & 0.18 & 0.77 & 0.22 & 2.11 & 0.30 & 0.96 & 0.69 & 1.23 & 1.03 & 0.78 & 0.42 & 0.74 & 0.37 \\
\hline $05 / 13$ & & & & & & & 0.73 & 0.42 & 1.16 & 0.17 & 1.15 & 1.19 & 1.99 & 0.18 & 0.63 & 0.18 & 2.30 & 0.30 & 1.17 & 0.94 & 1.38 & 1.12 & 0.77 & 0.36 & 0.81 & 0.44 \\
\hline $06 / 13$ & & & & & & & 0.54 & 0.40 & 0.90 & 0.11 & 2.22 & 1.38 & 2.89 & 0.16 & 0.77 & 0.19 & 2.02 & 0.20 & 1.28 & 1.03 & 1.46 & 0.81 & 0.79 & 0.38 & 0.76 & 0.52 \\
\hline $07 / 13$ & & & & & & & 0.72 & 0.54 & & & 1.51 & 1.56 & 1.42 & 0.25 & 0.76 & 0.23 & 1.79 & 0.14 & 1.37 & 1.09 & 1.35 & 1.10 & 0.75 & 0.36 & 0.91 & 0.59 \\
\hline $08 / 13$ & & & & & 0.83 & 0.07 & 1.01 & 0.30 & 1.60 & 0.31 & 0.60 & 0.35 & 2.25 & 0.10 & 0.80 & 0.20 & 1.43 & 0.12 & 1.48 & 1.18 & 1.44 & 1.03 & 0.83 & 0.30 & 1.07 & 0.49 \\
\hline $09 / 13$ & & & & & & & & & 1.12 & 0.26 & 0.92 & 0.81 & 2.50 & 0.08 & 0.66 & 0.27 & 1.59 & 0.17 & 1.32 & 1.35 & 1.56 & 1.01 & 0.36 & 0.63 & 0.60 & 0.81 \\
\hline
\end{tabular}

Durante el periodo de monitoreo septiembre 2012 - septiembre 2013 los datos obtenidos durante este lapso de tiempo arrojaron los siguientes resultados:

- Especie aliso: la estaca, presenta un crecimiento del diámetro de 0.97 centímetros y longitud de 0.49 metros, mientras que el brote, el crecimiento del diámetro es de 0.50 centímetros y longitud de 0.31 metros.

- El crecimiento del diámetro de la especie Polylepis sp. registrado es 0.93 centímetros para la estaca y 0.66 centímetros para el brote, mientras que la longitud muestra un crecimiento de 0.48 metros para la estaca y 0.32 metros para el brote.

- Especie chilca: la estaca, presenta un crecimiento del diámetro de 1.03 centímetros y longitud de 0.37 metros, mientras que el brote, el crecimiento del diámetro es de 1.03 centímetros y longitud de 0.78 metros.

- El crecimiento del diámetro de la especie lechero registrado es 2.04 centímetros para la estaca y 0.75 centímetros para el brote, mientras que la longitud muestra un crecimiento de 0.32 metros para la estaca y 0.22 metros para el brote.

- Especie tilo: la estaca, presenta un crecimiento del diámetro de 1.93 centímetros y longitud de 0.47 metros, mientras que el brote, el crecimiento del diámetro es de 1.02 centímetros y longitud de 0.81 metros. 
- La especie pusupato presenta los siguientes resultados: pusupatos parados tienen un diámetro de 1.34 centímetros y longitud de 1.03 metros; los pusupatos acostados, la estaca tiene un diámetro de 0.85 centímetros y longitud de 0.44 metros, el brote tiene un diámetro de 0.71 centímetros y longitud de 0.42 metros.

\begin{tabular}{|c|c|c|c|c|c|c|c|c|c|c|c|c|c|c|c|c|c|c|c|c|c|c|c|c|c|c|}
\hline \multirow{3}{*}{$\bigcup_{\text {Mes }}$} & \multicolumn{4}{|c|}{ Aliso } & \multicolumn{4}{|c|}{ Polylepis } & \multicolumn{4}{|c|}{ Chilca } & \multicolumn{4}{|c|}{ Lechero } & \multicolumn{4}{|c|}{ Tilo } & \multirow{2}{*}{\multicolumn{2}{|c|}{$\begin{array}{c}\begin{array}{c}\text { Pusupato } \\
\text { (parados) }\end{array} \\
\text { Estaca }\end{array}$}} & \multicolumn{4}{|c|}{$\begin{array}{l}\text { Pusupato } \\
\text { (acostados) }\end{array}$} \\
\hline & \multicolumn{2}{|c|}{ Estaca } & \multicolumn{2}{|c|}{ Brote } & \multicolumn{2}{|c|}{ Estaca } & \multicolumn{2}{|c|}{ Brote } & \multicolumn{2}{|c|}{ Estaca } & \multicolumn{2}{|c|}{ Brote } & \multicolumn{2}{|c|}{ Estaca } & \multicolumn{2}{|c|}{ Brote } & \multicolumn{2}{|c|}{ Estaca } & \multicolumn{2}{|c|}{ Brote } & & & \multicolumn{2}{|c|}{ Estaca } & \multicolumn{2}{|c|}{ Brote } \\
\hline & $\mathrm{D}$ & $\mathrm{L}$ & $\mathrm{D}$ & $\mathrm{L}$ & $\mathrm{D}$ & $\mathrm{L}$ & $\mathrm{D}$ & $\mathrm{L}$ & D & $\mathrm{L}$ & $\mathrm{D}$ & $\mathrm{L}$ & $\mathrm{D}$ & $\mathrm{L}$ & $\mathrm{D}$ & $\mathrm{L}$ & $\mathrm{D}$ & $\mathrm{L}$ & $\mathrm{D}$ & $\mathrm{L}$ & $\mathrm{D}$ & $\mathrm{L}$ & $\mathrm{D}$ & I & $\mathrm{D}$ & $\mathrm{L}$ \\
\hline $09 / 12$ & 0.51 & 0.41 & & & 0.52 & 0.40 & & & 0.45 & 0.42 & & & 1.08 & 0.32 & & & 0.96 & 0.62 & & & 0.14 & 0.11 & 0.26 & 0. & & \\
\hline $10 / 12$ & 0.20 & 0.10 & & & 0.31 & 0.11 & & & 0.25 & 0.10 & & & 1.67 & 0.38 & & & 0.51 & 0.00 & & & 0.30 & 0.21 & 0.13 & 0. & & \\
\hline $11 / 12$ & 0.25 & 0.73 & & & 0.32 & 0.13 & & & 0.18 & 0.06 & & & 0.43 & 0.13 & & & 1.07 & 0.11 & & & 0.31 & 0.17 & 0.13 & 0. & & \\
\hline $12 / 12$ & 0.23 & 0.06 & 0.16 & 0.51 & 0.20 & 0.12 & \multicolumn{2}{|c|}{0.18} & 0.30 & 0.25 & 0.11 & 0.09 & 0.57 & 0.12 & 0.27 & 0.08 & 1.18 & 0.11 & 0.24 & 0.31 & 0.25 & 0.16 & 0.22 & 0 . & 0.12 & 0.07 \\
\hline $01 / 13$ & 0.27 & 0.05 & 0.16 & 0.08 & 0.11 & 0.12 & 0.14 & 0.02 & 0.21 & 0.08 & 0.31 & 0.20 & 0.53 & 0.10 & 0.30 & 0.19 & 1.11 & 0.15 & 0.28 & 0.24 & 0.24 & 0.26 & 0.20 & 0. & 0.16 & 0.10 \\
\hline $02 / 13$ & 0.20 & 0.04 & 0.58 & 0.15 & 0.11 & 0.05 & 0.11 & 0.05 & 0.19 & 0.07 & 0.55 & 0.34 & 0.74 & 0.12 & 0.38 & 0.16 & 1.13 & 0.26 & 0.40 & 0.28 & \multicolumn{2}{|c|}{0.23} & 0.43 & 0 . & 0.23 & 0.15 \\
\hline $03 / 13$ & 0.30 & 0.04 & & & 0.05 & 0.02 & 0.56 & 0.03 & 0.47 & 0.02 & 0.13 & 0.08 & 0.71 & 0.17 & 0.31 & 0.12 & 1.23 & 0.10 & 0.39 & 0.34 & 0.26 & 0.25 & 0.11 & 0 . & 0.12 & 0.09 \\
\hline $04 / 13$ & \multicolumn{2}{|c|}{0.00} & & & & & \multicolumn{2}{|c|}{0.00} & 0.58 & 0.01 & 0.69 & 0.04 & 0.88 & 0.10 & 0.24 & 0.14 & 0.91 & 0.31 & 0.66 & 0.48 & 0.27 & 0.24 & 0.21 & 0. & 0.14 & 0.10 \\
\hline $05 / 13$ & & & & & & & \multicolumn{2}{|c|}{0.04} & 0.18 & 0.06 & 0.24 & 0.21 & 0.47 & 0.12 & 0.21 & 0.11 & 1.15 & 0.34 & 0.70 & 0.60 & 0.28 & 0.24 & 0.12 & 0. & 0.22 & 0.12 \\
\hline $06 / 13$ & & & & & & & 0.16 & 0.05 & 0.32 & 0.05 & 1.01 & 0.14 & 0.78 & 0.04 & 0.09 & 0.11 & 0.85 & 0.14 & 0.81 & 0.67 & 0.35 & 0.43 & 0.17 & 0 . & 0.28 & 0.12 \\
\hline $07 / 13$ & & & & & & & \multicolumn{2}{|c|}{0.00} & & & 0.34 & 0.42 & 0.83 & 0.19 & 0.43 & 0.14 & 1.18 & 0.06 & 0.82 & 0.75 & 0.32 & 0.23 & 0.12 & 0 . & 0.22 & 0.18 \\
\hline $08 / 13$ & & & & & \multicolumn{2}{|c|}{0.00} & & & 0.20 & 0.17 & 0.10 & 0.35 & 0.83 & 0.05 & 0.38 & 0.11 & 0.13 & 0.02 & 1.04 & 0.77 & 0.34 & 0.25 & 0.12 & 0 . & 0.22 & 0.08 \\
\hline $09 / 13$ & & & & & & & & & 0.13 & 0.08 & 0.47 & 0.41 & 0.61 & 0.06 & 0.26 & 0.10 & & & 0.93 & 0.79 & 0.40 & 0.29 & 0.13 & 0. & 0.10 & 0.24 \\
\hline
\end{tabular}

Tabla 4 Desviación estándar de estacas y brotes de las especies utilizadas. $\mathrm{D}=$ diámetro en centímetros $\mathrm{L}=$ Longitud en metros

Durante el periodo de monitoreo septiembre 2012 - septiembre 2013 los datos obtenidos durante este lapso de tiempo arrojaron los siguientes resultados:

- Especie aliso: la estaca, presenta un crecimiento del diámetro de 0.25 centímetros y longitud de 0.18 metros, mientras que el brote, el crecimiento del diámetro es de 0.30 centímetros y longitud de 0.25 metros.

- El crecimiento del diámetro de la especie Polylepis sp. registrado es 0.20 centímetros para la estaca y 0.13 centímetros para el brote, mientras que la longitud muestra un crecimiento de 0.12 metros para la estaca y 0.04 metros para el brote.

- Especie chilca: la estaca, presenta un crecimiento del diámetro de 0.28 centímetros y longitud de 0.11 metros, mientras que el brote, el crecimiento del diámetro es de 0.40 centímetros y longitud de 0.23 metros.

- El crecimiento del diámetro de la especie lechero registrado es 0.78 centímetros para la estaca y 0.29 centímetros para el brote, mientras que la longitud muestra un crecimiento de 0.15 metros para la estaca y 0.13 metros para el brote. 
- Especie tilo: la estaca, presenta un crecimiento del diámetro de 0.88 centímetros y longitud de 0.17 metros, mientras que el brote, el crecimiento del diámetro es de 0.63 centímetros y longitud de 0.52 metros.

- La especie pusupato presenta los siguientes resultados: pusupatos parados tienen un diámetro de 0.28 centímetros y longitud de 0.24 metros; los pusupatos acostados, la estaca tiene un diámetro de 0.18 centímetros y longitud de 0.14 metros, el brote tiene un diámetro de 0.18 centímetros y longitud de 0.13 metros.

\section{EI Recreo}

\begin{tabular}{|c|c|c|c|c|c|c|c|c|c|c|c|c|}
\hline \multirow{3}{*}{ Mes Especie } & \multicolumn{4}{|c|}{ Aliso } & \multicolumn{4}{|c|}{ Polylepis } & \multicolumn{4}{|c|}{ Chilca } \\
\hline & \multicolumn{2}{|c|}{ Estaca } & \multicolumn{2}{|c|}{ Brote } & \multicolumn{2}{|c|}{ Estaca } & \multicolumn{2}{|c|}{ Brote } & \multicolumn{2}{|c|}{ Estaca } & \multicolumn{2}{|c|}{ Brote } \\
\hline & $\mathrm{D}$ & $\mathrm{L}$ & $\mathrm{D}$ & $\mathrm{L}$ & $\mathrm{D}$ & $\mathrm{L}$ & $\mathrm{D}$ & $\mathrm{L}$ & $\mathrm{D}$ & $\mathrm{L}$ & $\mathrm{D}$ & $\mathrm{L}$ \\
\hline $09 / 12$ & 1.39 & 1.68 & & & 0.83 & 1.91 & & & 1.25 & 0.30 & & \\
\hline $10 / 12$ & 0.96 & 0.26 & 0.44 & 0.15 & 0.64 & 0.29 & 0.42 & 0.17 & 1.35 & 0.24 & 0.37 & 0.14 \\
\hline $11 / 12$ & 0.80 & 0.23 & 0.46 & 0.05 & 0.64 & 0.31 & 0.45 & 0.08 & 1.31 & 0.25 & 0.39 & 0.12 \\
\hline $12 / 12$ & 0.06 & 0.21 & 0.03 & 0.05 & 0.08 & 0.28 & 0.05 & 0.14 & 0.93 & 0.25 & \multicolumn{2}{|c|}{0.22} \\
\hline $01 / 13$ & 0.68 & 0.17 & 0.45 & 0.30 & 0.68 & 0.25 & 0.47 & 0.17 & 1.35 & 0.29 & 0.51 & 0.35 \\
\hline $02 / 13$ & 0.76 & 0.14 & 0.37 & 0.05 & 0.73 & 0.17 & 0.43 & 0.09 & 1.25 & 0.23 & 0.56 & 0.45 \\
\hline $03 / 13$ & 0.72 & 0.12 & 0.37 & 0.07 & 0.74 & 0.19 & 0.43 & 0.14 & 1.26 & 0.25 & 0.56 & 0.55 \\
\hline $04 / 13$ & 0.09 & 0.10 & 0.05 & 0.06 & 0.09 & 0.14 & 0.04 & 0.08 & 1.35 & 0.28 & 0.42 & 0.60 \\
\hline $05 / 13$ & & & & & 0.75 & 0.27 & 0.64 & 0.10 & 1.53 & 0.34 & 0.69 & 0.67 \\
\hline $06 / 13$ & 0.90 & 0.22 & & & 0.73 & 0.03 & 0.39 & 0.06 & 1.78 & 0.23 & 0.70 & 0.68 \\
\hline $07 / 13$ & & & & & 0.27 & 0.29 & 0.30 & 0.11 & 1.37 & 0.25 & 0.59 & 0.67 \\
\hline $08 / 13$ & & & & & 0.09 & 0.10 & 0.08 & 0.07 & 0.66 & 0.23 & 0.31 & 0.66 \\
\hline
\end{tabular}

Tabla 5 Media de estacas y brotes de las especies utilizadas. $\mathrm{D}=$ diámetro en centímetros $\mathrm{L}=\mathrm{L}$.

Durante el periodo de monitoreo septiembre 2012 - septiembre 2013 los datos obtenidos durante este lapso de tiempo arrojaron los siguientes resultados:

- Especie aliso: la estaca, presenta un crecimiento del diámetro de 0.71 centímetros y longitud de 0.35 metros, mientras que el brote, el crecimiento del diámetro es de 0.31 centímetros y longitud de 0.10 metros.

- El crecimiento del diámetro de la especie Polylepis sp. registrado es 0.52 centímetros para la estaca y 0.34 centímetros para el brote, mientras que la longitud muestra un crecimiento de 0.35 metros para la estaca y 0.11 metros para el brote.

- Especie chilca: la estaca, presenta un crecimiento del diámetro de 1.28 centímetros y longitud de 0.29 metros, mientras que el brote, el crecimiento del diámetro es de 0.48 centímetros y longitud de 0.46 metros.

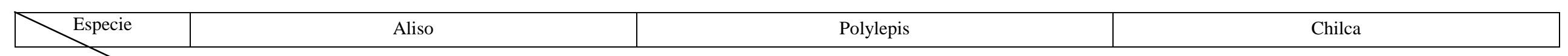




\begin{tabular}{|c|c|c|c|c|c|c|c|c|c|c|c|c|}
\hline \multirow[b]{2}{*}{ Mes } & \multicolumn{2}{|c|}{ Estaca } & \multicolumn{2}{|c|}{ Brote } & \multicolumn{2}{|c|}{ Estaca } & \multicolumn{2}{|c|}{ Brote } & \multicolumn{2}{|c|}{ Estaca } & \multicolumn{2}{|c|}{ Brote } \\
\hline & $\mathrm{D}$ & $\mathrm{L}$ & $\mathrm{D}$ & $\mathrm{L}$ & $\mathrm{D}$ & $\mathrm{L}$ & $\mathrm{D}$ & $\mathrm{L}$ & $\mathrm{D}$ & $\mathrm{L}$ & $\mathrm{D}$ & $\mathrm{L}$ \\
\hline $10 / 12$ & 0.71 & 0.35 & & & 0.37 & 0.28 & & & 0.65 & 0.17 & & \\
\hline $11 / 12$ & 0.64 & 0.15 & 0.13 & 0.06 & 0.22 & 0.14 & 0.17 & 0.12 & 1.13 & 0.15 & 0.12 & 0.09 \\
\hline $12 / 12$ & 0.39 & 0.13 & 0.15 & 0.04 & 0.17 & 0.18 & 0.12 & 0.03 & 0.61 & 0.15 & 0.16 & 0.09 \\
\hline $01 / 13$ & 0.02 & 0.07 & \multicolumn{2}{|c|}{0.01} & 0.10 & 0.15 & \multicolumn{2}{|c|}{0.07} & 0.78 & 0.15 & 0.00 & 1.27 \\
\hline $02 / 13$ & 0.19 & 0.08 & 0.16 & 0.32 & 0.22 & 0.16 & 0.17 & 0.16 & 0.98 & 0.21 & 0.27 & 0.19 \\
\hline $03 / 13$ & 0.10 & 0.07 & \multicolumn{2}{|c|}{0.00} & 0.35 & 0.07 & 0.14 & 0.04 & 0.56 & 0.19 & 0.23 & 0.19 \\
\hline $04 / 13$ & 0.14 & 0.06 & 0.06 & 0.04 & 0.31 & 0.10 & 0.14 & 0.30 & 0.85 & 0.16 & 0.75 & 0.21 \\
\hline $05 / 13$ & 0.02 & 0.06 & 0.02 & 0.01 & 0.02 & 0.04 & \multicolumn{2}{|c|}{0.02} & 1.08 & 0.24 & 0.36 & 0.27 \\
\hline $06 / 13$ & & & & & 0.05 & 0.17 & 0.13 & 0.02 & 1.20 & 0.32 & 0.30 & 0.32 \\
\hline $07 / 13$ & & & & & 0.26 & 0.02 & 0.10 & 0.03 & 0.78 & 0.08 & 0.17 & 0.19 \\
\hline $08 / 13$ & & & & & 0.23 & 0.22 & 0.00 & 0.01 & 1.11 & 0.14 & 0.24 & 0.34 \\
\hline $09 / 13$ & & & & & \multicolumn{2}{|c|}{0.00} & 0.02 & 0.03 & 0.72 & 0.10 & 0.27 & 0.35 \\
\hline
\end{tabular}

Tabla 6 Desviación estándar de estacas y brotes de las especies utilizadas $\mathrm{D}=$ diámetro en centímetros L= Longitud en metros

Durante el periodo de monitoreo septiembre 2012 - septiembre 2013 los datos obtenidos durante este lapso de tiempo arrojaron los siguientes resultados:

- Especie aliso: la estaca, presenta un crecimiento del diámetro de 0.25 centímetros y longitud de 0.11 metros, mientras que el brote, el crecimiento del diámetro es de 0.08 centímetros y longitud de 0.07 metros.

- El crecimiento del diámetro de la especie Polylepis sp. registrado es 0.19 centímetros para la estaca y 0.10 centímetros para el brote, mientras que la longitud muestra un crecimiento de 0.13 metros para la estaca y 0.08 metros para el brote.

- Especie chilca: la estaca, presenta un crecimiento del diámetro de 0.87 centímetros y longitud de 0.17 metros, mientras que el brote, el crecimiento del diámetro es de 0.26 centímetros y longitud de 0.32 metros. 